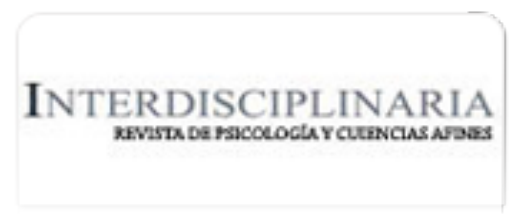

Interdisciplinaria

ISSN: 0325-8203

ISSN: 1668-7027

interdisciplinaria@fibercorp.com.ar

Centro Interamericano de Investigaciones Psicológicas y

Ciencias Afines

Argentina

Silenzi, María Inés

El problema de marco y el debate sobre racionalidad humana en psicología cognitiva: Racionalidad estándar vs. Racionalidad ecológica

Interdisciplinaria, vol. 35, núm. 2, 2018, Julio-, pp. 445-457

Centro Interamericano de Investigaciones Psicológicas y Ciencias Afines

Argentina

Disponible en: https://www.redalyc.org/articulo.oa?id=18058785001

Cómo citar el artículo

Número completo

Más información del artículo

Página de la revista en redalyc.org

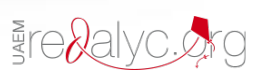

Sistema de Información Científica Redalyc Red de Revistas Científicas de América Latina y el Caribe, España y Portugal Proyecto académico sin fines de lucro, desarrollado bajo la iniciativa de acceso abierto 


\title{
El problema de marco y el debate sobre racionalidad humana en psicología cognitiva: Racionalidạd estándar vs. Racionalidad ecológica
}

\section{The frame problem and the debate on human rationality in cognitive psychology: Standard rationality vs. Ecological rationality}

\author{
María Inés Silenzi*
}

\begin{abstract}
*Doctora en Filosofía. Jefa de Tabajos Prácticos en la materia Epistemología y Metodología de la Investigación, Universidad Nacional del Sur. Investigadora Asistente del Consejo Nacional de Investigaciones Científicas y Técnicas (CONICET).E-mail: misilenzi@uns.edu.ar
\end{abstract}

Instituto de Investigaciones Económicas y Sociales del Sur (IIESS). Consejo Nacional de Investigaciones Científicas y Técnicas (CONICET). Buenos Aires, Argentina.

Universidad Nacional del Sur (UNS). Bahía Blanca, Argentina.

\begin{abstract}
Resumen
El problema de marco, entendido como un problema de relevancia, es uno de los principales problemas de las Ciencias Cognitivas. Frente a algunos autores que lo consideran aún un problema pendiente de solución, se ha propuesto que una de las posibles soluciones es el uso de heurísticas. El inconveniente es que hay dos programas de investigación sobre heurísticas: el programa de Heurísticas y Sesgos y el de Heurísticas Rápidas y Frugales. Estos programas se han enfrentado en un gran debate acerca de la racionalidad humana postulando distintas concepciones de racionalidad. Así, el objetivo del presente trabajo fue indagar cómo se relaciona el problema de marco con tal debate sobre racionalidad. Se argumenta que el fenómeno fundamental del problema de marco está mejor articulado a una concepción optimista de la racionalidad humana y sería más cercano a la concepción de racionalidad ecológica propuesta por el programa de Heurísticas Rápidas y Frugales.
\end{abstract}

Palabras clave: Problema de marco; Heurísticas; Racionalidad; Racionalidad Estándar; Racionalidad Ecológica.

\begin{abstract}
The frame problem (Mc Carthy \& Hayes, 1969) arose within the field of Artificial Intelligence research, more precisely within the calculation of situations, as the problem about how to represent, in the most succinct way possible, the fact that in a particular situation most properties of objects remain the same or are not disturbed by the performance of a particular action. Despite its logical origin, it did not take long for this problem to be addressed by several philosophers becoming the subject of major discussions that still surround the frame problem. One of the key difficulties that this problem tries to solve is the so-called difficulty of the vastness of the information: it would seem impossible to analyze each of the pieces of information from a vast set of information and evaluate, in each case, if it is or not relevant to carry out the task in question. Of course, exploring a vast amount of information with very limited cognitive and temporal resources is one of the main computational limitations that the frame problem must overcome.

Currently, the resolution of the frame problem has awakened a renewed interest in recent years, and its resolution has been taken up again.
\end{abstract}


Proof of this is Fodor's (2000) treatment of the frame problem by criticizing some current strategies for its solution, such as the use of heuristics. The use of heuristics as strategies to explain cognitive processes is a subject of great relevance at present and especially of great interest in the area of psychology. Generally, the heuristics understood, prima facie, as practical rules that, with little information, offer reasonable solutions to problems or questions would solve the computational limitations of the frame problem by providing the computational processing sought. It must be considered that, as the difficulty of the vastness of information reflects, the determination of relevance is not computationally easy since it is necessary to evaluate large amounts of information and that is why the use of heuristics would appear as the main candidate to limit the computational load in the determination of relevance.

The problem is that there are two research programs that work on heuristics: the Heuristics and Biases program and the Heuristics Fast and Frugal program. In literature of the area, the rivalry between these programs with respect to the methodology and way of working is often highlighted, but also in terms of the scope and limits of the rationality that each of these programs postulates. More particularly, both programs have faced a great debate about human rationality, postulating different conceptions of rationality. Taking into account that it has been proposed to interpret the frame problem as a problem of rationality, the general objective of this paper is to investigate how the frame problem relates to such a debate on rationality; more specifically, it will be questioned which of these conceptions of rationality would be better articulated with the fundamental phenomenon of the frame problem

First, in this work we will analyze, the standard conception of rationality, considering some o the main criticisms that it has faced. These objections will allow us to introduce later the ecological conception of rationality postulated by the program of Fast and Frugal Heuristics. Finally, it will be evaluated which of these two concepts would be more linked to the fundamental phenomenon of the frame problem, in order to finally present the conclusions. We argue that the fundamental phenomenon of the frame problem is better articulated to an optimistic view of human rationality and it would be closer to the concept of ecological rationality proposed by the Heuristic Fast and Frugal program.

Key words: Frame Problem; Heuristics; Rationality; Ecological Rationality; Standard Rationality.

\section{Introducción}

El problema de marco (Mc Carthy \& Hayes, 1969) surgió dentro del campo de investigación de la Inteligencia Artificial, más precisamente dentro del cálculo de situaciones, como el problema acerca de cómo representar, de la forma más sucinta posible, el hecho de que en una situación particular la mayoría de las propiedades de los objetos permanecen iguales o no son perturbadas por la realización de una determinada acción (Shanahan, 1997). Pese a su origen lógico, no pasó mucho tiempo para que este problema fuera abordado por varios filósofos (véase Brown, 1987; Dennett, 1984; Fodor, 1986; Ford \& Hayes, 1991; Ford \& Pylyshyn, 1996 y Pylyshyn, 1980, entre otros), convirtiéndose en motivo de grandes discusiones que aún rodean al problema de marco. Uno de los grandes debates que todavía se discute gira en torno a su dificultad definicional y resolutiva. No es el propósito de este trabajo enfocarse detalladamente en cada una de estas dificultades; simplemente se busca enfatizar la relación que se establece entre ambas dificultades: antes de evaluar cualquier solución que intente resolver el problema de marco, es necesario aclarar anteriormente la interpretación específica que de éste problema se tenga en mente.

La dificultad definicional del problema de marco surge como consecuencia de las múltiples definiciones que se han propuesto (Morgenstern, 1996; Stein, 1990). Se interpretará el problema de marco como refiriendo a un conjunto de problemas (aspectos) que tienen que ver con la manera en que 
un agente selecciona la información relevante, de entre un vasto conjunto de información, de manera tal que logra realizar la tarea con cierto éxito o eficientemente. En la literatura del área suele aceptarse que a las personas parece venirle a la mente solamente la información relevante y en el momento preciso y de manera adecuada; a este fenómeno se lo llamará de ahora en más el fenómeno fundamental del problema de marco. Este fenómeno, claro está, da cuenta de que el problema de marco puede ser considerado como un problema de racionalidad. Esta interpretación será de sumo interés en este trabajo: más específicamente, y en virtud de considerar en este trabajo el problema de marco como un problema de racionalidad, es que se analizarán distintas concepciones de racionalidad.

Continuando con esta descripción del problema de marco, una de las dificultades claves que éste intenta resolver, es la denominada dificultad de la vastedad de la información: parecería imposible analizar cada una de las piezas de información de entre un vasto conjunto de información y evaluar, en cada uno de los casos, si es o no relevante para llevar a cabo la tarea en cuestión. Claro está que explorar vasta cantidad de información con recursos cognitivos y temporales muy limitados es una de las principales limitaciones computacionales que el problema de marco debe superar.

Actualmente, la resolución del problema de marco ha despertado un renovado interés en los últimos años, siendo así retomada su dificultad resolutiva. Muestra de ello es el tratamiento que Fodor (2000) hace del problema de marco al criticar algunas estrategias actuales para su solución, como lo es el uso de las heurísticas. El uso de las heurísticas como estrategias para explicar los procesos cognitivos es un tema de investigación de gran vigencia en la actualidad y especialmente de mucho interés en el área de psicología (Carruthers, 2006, 2009; Gigerenzer \& Brighton, 2009).

Puntualmente, dos programas de investigación rivales en psicología cognitiva como lo son el de Heurísticas y Sesgos (li- derado por Daniel Kahneman) y el de Heurísticas Rápidas y Frugales (liderado por Gerd Gigerenzer) apelan al uso de heurísticas para explicar ciertas regularidades empíricas de razonamiento y decisión (véase Gilovich, Griffin \& Kahneman, 2002 y Gigerenzer \& Selten, 2001, entre otros). De manera general, las heurísticas entendidas, prima facie, como reglas prácticas que, con poca información, ofrecen soluciones razonables a problemas o preguntas, resolverían las limitaciones computacionales del problema de marco al proveer la tratabilidad computacional buscada. Hay que considerar que, como lo refleja la dificultad de la vastedad de información, la determinación de la relevancia no es computacionalmente fácil, pues se requiere evaluar grandes cantidades de información y es por ello que el uso de heurísticas aparecería como el principal candidato para limitar la carga computacional en la determinación de la relevancia (Samuels, 2010).

No obstante, en literatura del área, suele destacarse la rivalidad de entre estos programas con respecto a la metodología y forma de trabajo, pero también en cuanto a los alcances y límites de la racionalidad que cada uno de estos programas postula. Más particularmente ambos programas se han enfrentado en un gran debate acerca de la racionalidad humana, postulando concepciones diferentes de racionalidad. Teniendo en cuenta que se ha propuesto interpretar el problema de marco como un problema de la racionalidad, el objetivo general de este trabajo es indagar cómo se relaciona el problema de marco con tal debate sobre racionalidad; más concretamente se cuestionará cuál de estas concepciones de racionalidad se articularía mejor con el fenómeno fundamental del problema de marco. Se argumentará que el fenómeno fundamental del problema de marco está ligado a una concepción optimista de la racionalidad humana y estaría mejor articulado con la concepción de racionalidad ecológica propuesta por el programa de Heurísticas Rápidas y Frugales. 
En primera instancia, se propondrá la interpretación del problema de marco de interés en este trabajo, atendiendo a lo que se denomina el fenómeno fundamental del problema de marco. Luego se analizará, la concepción estándar de racionalidad, considerando algunas de las principales críticas a las que se ha enfrentado. Estas objeciones nos permitirán introducir luego la concepción de racionalidad ecológica postulada por el programa de Heurísticas Rápidas y Frugales. Por último, y atendiendo a la disputa de interés en este trabajo, se evaluará cuál de ambas concepciones se encontraría más ligada al fenómeno fundamental del problema de marco, para presentar finalmente las conclusiones.

\section{El fenómeno fundamental del problema de marco}

Pese a la ya mencionada dificultad de definir el problema de marco, en algunos trabajos (véase por ejemplo Silenzi, 2015) se argumenta que se puede encontrar cierta tendencia entre las distintas definiciones del problema a interpretarlo finalmente como un conjunto de problemas relacionados con la determinación de relevancia (Crockett, 1994; Dreyfus, 1979; Glymour, 1987; Ludwing \& Schneider, 2008; Pinker, 2005; Schneider, 2007; Shanahan, 2009, entre otros). No se atenderá en este trabajo a los distintos aspectos del problema (véase una clasificación de éstos en Silenzi, 2014) aunque sí se tendrá en cuenta cierta regularidad exhibida por los seres humanos a la hora de determinar relevancia. En la literatura del área se da por supuesto que las personas suelen determinar qué es relevante para una tarea dada de manera rápida y adecuada, aunque de vez en cuando surjan errores. Esta regularidad, denominada fenómeno fundamental del problema de marco, permite interpretarlo, de ahora en más, como un problema de racionalidad.

No parece plausible atribuirle al azar la prontitud y el nivel de éxito logrado y, por lo tanto, cualquier noción de racionalidad asociada a este aspecto del problema de marco (psicológico), debería dar cuenta del mencionado fenómeno fundamental; más específicamente, una concepción de racionalidad vinculada a este aspecto debería dar cuenta de cómo a las personas parece venirles a la mente la información relevante para una tarea de entre una cantidad desmesurada de información que poseen y eso, además, en el momento preciso y de manera adecuada.

Parece apropiado ilustrar el fenómeno fundamental del problema de marco a través de uno de los ejemplos más representativos y más claros que es el que presenta Dennett (1984) cuyo título suele traducirse como Las ruedas del conocimiento: el problema de marco en la Inteligencia Artificial. Este ejemplo ha sido descripto en numerosos trabajos a la hora de introducir el problema de marco, puesto que es clave en la comprensión de las divergencias entre el modo en que los seres humanos resolvemos el problema de marco y la (supuesta) imposibilidad de ser resuelto por agentes artificiales. Más particularmente, el autor introduce una situación en la que se enfatizan ciertos aprietos en los que se encuentra un agente artificial al enfrentarse al problema de marco. En este particular ejemplo se apela a un experimento mental en el que se invita a imaginarnos un robot $\mathrm{R} 1$ al que sus diseñadores le fijan la tarea de recuperar su batería de repuesto. La batería se encuentra dentro de un carro en una habitación cerrada en la que hay una bomba programada para estallar en poco tiempo. R1 hace la hipótesis de que una cierta acción, sacar (Carro, Habitación) le permitiría cumplir su tarea. Entra pues a la habitación y saca el carro con su batería de repuesto. Desafortunadamente, al estar la bomba también dentro del carro, el robot estalla a los pocos segundos.

Los diseñadores de R1, para evitar tal explosión, creen que es necesario que el robot no sólo considere las implicaciones intencionadas de sus actos, sino también las implicaciones secundarias. Así, diseñan el siguiente robot, llamado R1D1, que también encuentra la batería y diseña un plan de acción; sin embargo, cuando acaba de deducir 
que quitar el carro hacia afuera de la habitación, no haría cambiar el color de las paredes y está embarcándose en la comprobación de la siguiente implicación, la bomba explota.

Con esta situación, los diseñadores consideran necesario enseñarle al robot a diferenciar, a través de mecanismos de inferencia, aquellas implicaciones que son pertinentes y que deben tenerse en cuenta al diseñar un plan de acción, de aquellas otras que no lo son. El diferenciar tales implicaciones permitiría que el robot no se quede congelado considerando todas y cada una de las implicaciones, relevantes e irrelevantes. Llaman a este tercer robot R2D1 y le dan la misma orden. Cuando el robot localiza la batería, se sienta afuera de la habitación. Los diseñadores, angustiados por ver que el robot se queda congelado de nuevo, le gritan que haga algo y el robot responde: "¡Lo estoy haciendo (...) estoy ignorando diligentemente las miles de implicaciones que determiné improcedentes! ¡Tan pronto descubra una implicación improcedente, la sumo a la lista de las que debo ignorar!" Así, mientras el robot se concentra en omitir consideraciones innecesarias, explota la bomba y el robot se queda sin batería.

Ahora bien, Dennett parece asumir que ninguno de los tres robots ha solucionado exitosamente el problema de marco. El primer robot no tiene un mecanismo que le permita considerar de manera completa las implicaciones de sus actos. El segundo robot tiene esta capacidad, pero no la de distinguir las consecuencias relevantes de las irrelevantes. Y finalmente, el tercero posee esta última capacidad, pero carece de la habilidad de coordinar esa distinción con las acciones necesarias para la tarea, al menos, en un tiempo prudencial. Así, se puede argumentar que Dennett está comparando el desempeño ineficiente de estos robots con la aparente eficiencia humana al momento de determinar relevancia. La pregunta que parece tener en mente es ¿cómo es que al momento de realizar una determinada tarea, y dada la vasta cantidad de información disponible, los seres humanos somos capaces la mayoría de las veces (contrariamente a un agente artificial) de determinar relevancia exitosamente? Esta pregunta es la que subyace al fenómeno fundamental del problema de marco y la que cualquier resolución del problema de marco debe intentar resolver.

De acuerdo con el ejemplo de los robots de Dennett, pareciera que un robot determinaría relevancia exitosamente (suponiendo poder atribuirle ciertas habilidades humanas), si lograra desactivar la bomba luego de generar expeditivamente una secuencia de acciones que no lo terminen paralizando computacionalmente, o de otra manera, si lograra llevar a cabo la tarea. A la luz de lo examinado, se considerará que es requisito necesario, en lo que respecta a aquella noción de racionalidad vinculada al fenómeno fundamental del problema de marco, que ésta explicite cómo es que los seres humanos, a pesar de las limitaciones a las que irremediablemente debemos enfrentarnos, logramos determinar relevancia exitosamente.

Visto resumidamente de qué trata el fenómeno fundamental del problema de marco, a continuación se plantea la disputa de interés en este trabajo: las distintas concepciones de racionalidad que plantean los dos programas de investigación sobre heurísticas. Se podría objetar el pasar por alto un examen detallado sobre el alcance de las heurísticas por resolver el problema de marco. Cabe aclarar que los alcances y limitaciones de las heurísticas para resolver o afrontar el problema de marco ya han sido expuestos en artículos anteriores. Por tanto, se dará por sentado que las heurísticas son apropiadas para solucionar al menos algunos de los aspectos del problema de marco, restringiéndo de ahora en más, solamente al debate sobre la racionalidad que de ésta cuestión se deriva.

\section{Distintas concepciones de racionalidad}

A continuación se describen las dos nociones de racionalidad que implícitamente sostienen cada uno de los programas de investigación examinados. Cabe 
aclarar que no se hará una descripción en detalle de estas concepciones, pues el presente trabajo se centra en evaluar las consecuencias que se derivan de cada una de ellas en lo que concierne al problema de marco interpretado como un problema de racionalidad.

\section{La concepción estándar de racionalidad}

El programa de Heurísticas y Sesgos iniciado por Tversky y Kahneman en los años 70 (véase un compendio en Gilovich, Griffin \& Kahneman, 2002) defiende actualmente el enfoque de procesamiento dual de información. Nuestras mentes serían procesadores de información, pero tendrían dos sistemas funcionando simultáneamente, en lugar de uno. El sistema 1 sería automático, requiere poco esfuerzo cognitivo, es de carácter asociativo, funcionamiento paralelo y procesamiento opaco. Por otra parte, habría un sistema 2 con las características opuestas: controlado, requiere mucho esfuerzo cognitivo, deductivo, serial, autoconsciente. Las heurísticas serían estrategias del sistema 1. Lo interesante es que este programa, para determinar -dada una situación de incertidumbre- si una respuesta es aceptable o errónea, suele usar como estándares normativos la lógica, el cálculo de probabilidades y la teoría de decisión (Tversky \& Kahneman, 1974). Con otras palabras, y de acuerdo a cierta normatividad previa, este programa presupone que se puede saber de antemano si una decisión resulta correcta o incorrecta: si el resultado se aleja o desvía del esperado, será considerado como un error; si, por el contrario, este mismo resultado se ajusta al esperable, entonces la misma decisión será considerada como correcta.

Es interesante destacar que estos investigadores evalúan las habilidades del razonamiento del agente dentro de laboratorios psicológicos (fuera del contexto real donde se encuentra el agente). Al estar en esta situación (artificial) no se considera la incidencia de ningún otro factor, como lo pueden ser las limitaciones temporales o computacionales, no alterándose en nada los resultados esperados. Por esta razón, Stein (1996) presupone que la concepción de racionalidad defendida por este programa es alguna versión de lo que él llama concepción estándar de racionalidad (the standard picture), puesto que ésta tampoco tiene en consideración posibles limitaciones humanas, como si los agentes dispusieran de tiempo y poder computacional infinitos. Sin duda, entre las ventajas que ofrece esta visión (Samuels, Stich \& Faucher, 2004) se destaca su universalidad, puesto que sus principios se aplican a todos los agentes racionales independientes del contexto donde éstos se encuentren, ofreciendo así estándares razonablemente precisos con los que evaluar el razonamiento humano. Los criterios del buen razonamiento se derivan de las reglas de la lógica deductiva, del cálculo de probabilidades y de las teorías formales de decisión, ofreciendo de esta manera, criterios precisos acerca de cómo se debería razonar.

Lo interesante es que frente a los resultados experimentales que muestran una gran variedad de sesgos, es decir, desviaciones de lo que se considera un buen razonamiento, algunos investigadores sacaron conclusiones pesimistas acerca de la racionalidad humana (conclusiones que más adelante se retomarán) aunque Tversky y Kahneman siempre se mantuvieron neutrales ante esta discusión (véase especialmente el prólogo de Gilovich, Griffin \& Kahneman, 2002). Más concretamente, estos autores proponen centrarse en los errores por una cuestión metodológica: al igual que en las ilusiones ópticas, en los sesgos se aprecia mejor el funcionamiento de los procesos de elaboración de juicios y decisiones. Así como se puede evaluar un juicio perceptivo recurriendo a ciertas leyes físicas independientes del sujeto que percibe, también se puede evaluar un razonamiento aplicando las reglas deductivas o el cálculo de probabilidades como criterios independientes de quien razona. Estas reglas funcionan a modo de estándar permitiendo saber cuando un juicio se aparta o no del razonamiento correcto. 
Es importante destacar para los propósitos de este estudio, la nula o escasa consideración que esta noción de racionalidad tiene del contexto a la hora de estimar si un agente determina exitosamente (o no) relevancia. Como ya se mencionó, el criterio normativo que adscribe a esta concepción es aplicado de antemano, sin que entre en consideración el agente in situ, es decir el agente adaptado al contexto en el que se encuentra. Tampoco tiene en cuenta las limitaciones temporales a las que debe adaptarse el ambiente, por lo que resulta suspendida para este programa cualquier cuestión relacionada con la adaptación del agente. El acople entre el agente y el contexto es omitido por los investigadores pertenecientes a este programa, puesto que evalúan nuestras habilidades cognitivas en situaciones artificiales sin estimar el éxito logrado (o no) en situaciones reales.

Precisamente uno de los debates más enriquecedores sobre racionalidad que destaca estas diferencias es aquel que contrapone la racionalidad entre un plano deontológico versus un plano consecuencialista. Si bien no se examinará este debate en profundidad, seguramente contribuiría a esclarecer las concepciones de racionalidad implícitas en ambos programas de investigación (Evans \& Over, 1996). Desde la perspectiva deontológica de la racionalidad, se considera que razonar correctamente es razonar exclusivamente de acuerdo a los estándares normativos que provee la lógica y el cálculo de probabilidades (Alexander \& Moore, 2008). La concepción de racionalidad estándar presupone esta perspectiva y en consecuencia, también el programa de Heurística y Sesgos. Por otro lado, y desde la perspectiva consecuencialista, razonar correctamente es hacerlo de manera tal que se logre alcanzar las metas u objetivos implicados en la tarea a llevar a cabo. En contraposición a la perspectiva deontológica, razonar de acuerdo con algún conjunto de reglas, sin tener en cuenta las limitaciones a las que se enfrenta el agente (dada la tarea encomendada y el objetivo implicado) no es constitutivo de un buen razonamiento. Te- niendo en cuenta estas diferencias se podrían clasificar de manera muy distinta las respuestas a las tareas planteadas por los investigadores de ambos programas. Desde una perspectiva deontológica, las respuestas a las tareas se podrían clasificar como correctas o no, mientras que desde la perspectiva consecuencialista, como resoluciones exitosas o no exitosas. Teniendo en cuenta que se está evaluando qué concepción de racionalidad se encuentra más ligada al fenómeno fundamental del problema de marco, que cuestiona cómo se selecciona aquella información que contribuye a satisfacer el objetivo de una tarea en cuestión, parecería que la concepción de racionalidad ecológica resulta más adecuada. Como se está examinando cuál concepción de racionalidad se asociaría mejor al problema de marco, se analizará esta última concepción en detalle, examinando sus alcances y limitaciones para con el fenómeno fundamental del problema de marco.

\section{La concepción ecológica de racionalidad}

El programa de Heurísticas Rápidas y Frugales ve los sesgos como artefactos de los procedimientos experimentales del programa rival y en consecuencia, tiene una postura relativamente optimista con respecto a la racionalidad humana (postura que también se retomará luego). Para estos investigadores existe evidencia empírica de que las inferencias llevadas a cabo por un agente, al enfrentarse a una tarea determinada, son, en contraposición al otro programa, dependientes de restricciones (o acotamientos) temporales y computacionales (Gigerenzer \& Selten, 2001). Con otras palabras, este programa sí toma en consideración los recursos limitados en tiempo, conocimiento y poder computacional del agente en cuestión. Pero, además de tener en cuenta estas limitaciones, defienden también que los ambientes tienen cierta estructura informacional, es decir, que éstos proveen cierta información acerca de algunos aspectos (aunque, crucialmente, no de to- 
dos) y que esta información es dada en un formato específico. Al concentrarse en estos puntos, este enfoque destaca en sus investigaciones el alcance y las limitaciones del acople entre heurísticas usadas por humanos y estructuras ambientales. Adquiriendo así particular importancia las estructuras del contexto, este programa defiende implícitamente una concepción de racionalidad distinta a la concepción estándar de racionalidad, pues tiene en consideración al agente inmerso en distintos contextos y enfrentado a posibles limitaciones de recursos temporales, informacionales, de poder computacional o de memoria, entre otros. Esta concepción de racionalidad es denominada por Gigerenzer, Czerlinski y Martingnon como racionalidad ecológica (2002). Los criterios de racionalidad usados por esta concepción son precisión, frugalidad y velocidad. La precisión se refiere al grado de correspondencia entre juicios o predicciones y la realidad; la frugalidad se refiere a la cantidad de información que una heurística dada necesita para operar. Finalmente, la velocidad se refiere a la manera en la cual la heurística dada, procesa la información (cuanto más simple sea este proceso, más rápido será el juicio o decisión).

Ahora bien, este programa de investigación difiere del anterior en un punto importante. Además de ofrecer un conjunto distinto de heurísticas, la posición global es que en general, las personas usan heurísticas adecuadas para resolver problemas, es decir, heurísticas que no tienden a generar errores sistemáticos. Es en este encuadre donde se pueden apreciar las diferencias con la concepción estándar de racionalidad: la concepción ecológica tiene en cuenta las limitaciones del agente y en consecuencia, toma en cuenta al agente in situ, es decir al agente inmerso en el contexto al que se enfrenta; la concepción de racionalidad estándar no tiene en cuenta ningún tipo de limitación y con ello, no considera al agente inmerso en ningún contexto posible. El contexto está determinado por la información disponible y por las tareas encomendadas. Estas divergencias son tomadas en cuenta por la concepción ecológica de la racionalidad: el contexto al que se enfrenta el agente resulta clave para estimar si el empleo de cierta heurística es adecuado o no, puesto que, de acuerdo a un contexto, cierta heurística puede llevar a resultados exitosos mientras que para otro contexto, no. Inclusive, habría contextos donde convendría usar las reglas formales de razonamiento y decisión.

En consecuencia, las estimaciones que ambas concepciones consideran a la hora de evaluar cómo se determina relevancia son muy distintas: de acuerdo a la concepción estándar de racionalidad éstas pueden resultar correctas o incorrectas mientras que de acuerdo a la concepción ecológica, éstas pueden resultar exitosas (eficientes) o no. Tal eficiencia lograda al momento de determinar relevancia, dada la tarea encomendada y la información disponible, se puede equipar al grado de éxito logrado con que la mayoría de las veces se determina relevancia, éxito que por otro lado, y volviendo al problema de interés en este trabajo, subyace al fenómeno fundamental del problema de marco. Cabe recordar que la regularidad que este fenómeno cuestiona es cómo la mayoría de las veces los seres humanos determinamos relevancia exitosamente. La concepción ecológica sería de más utilidad que la concepción estándar de racionalidad al exponer esta cuestión, puesto que prepondera la adaptación del agente al contexto, y con ello, el éxito obtenido al momento de determinar relevancia. Contrariamente, esta eficiencia resulta difícil de describir recurriendo a la concepción de racionalidad estándar, puesto que las reglas de inferencia a las que ésta suscribe, son independientes del contexto, resultando indistinto lograr o no con éxito la tarea encomendada. Estas divergencias permitirán abordar, aun más, cuál de ambas concepciones se encontraría mejor articulada al fenómeno fundamental del problema de marco. 


\section{Racionalidad optimista vs racionalidad pesimista}

La afirmación de que el programa de Heurísticas y Sesgos y el programa de Heurísticas Rápidas y Frugales son genuinamente rivales puede ponerse en duda. En particular, pueden ser concebidos como variantes de un solo programa difiriendo en las interpretaciones que éstos ofrecen a los efectos contextuales en razonamientos y toma de decisiones (Alfano, 2014; Fairweather \& Montemayor, 2014). Sin embargo, este trabajo se apoya en las propias declaraciones de los protagonistas, y en especial de Gigerenzer (1996), quien plantea su posición como en manifiesta oposición al programa de Heurísticas y Sesgos (véase también Gigerenzer, 2008). Ambos programas defienden la idea de que las personas utilizan heurísticas para elaborar juicios y tomar decisiones que involucran situaciones de incertidumbre y que la mayor ventaja, comparado con modelos normativos, es que las heurísticas reducen el tiempo y el esfuerzo para realizar juicios y decisiones. Pero, y retomando algunas de las discrepancias ya descriptas, el programa de Heurísticas y Sesgos sostiene que en determinadas circunstancias o contextos, estas heurísticas conducen a errores sistemáticos o sesgos. Por esta razón, estos investigadores apelan a las heurísticas cuando se encuentran errores sistemáticos, lo que revelaría cierta irracionalidad particularmente en aquellos casos que se alejan de las leyes de la probabilidad (Gigerenzer, Todd \& ABC Research Group, 1999). En consecuencia, la perspectiva de nuestra racionalidad que este programa defiende es un tanto pesimista en comparación con la visión optimista de la racionalidad que defiende el programa de investigación rival. En efecto, si bien Gigerenzer y sus colaboradores (2002) concuerdan con el hecho que las personas utilizan heurísticas para razonamientos y decisiones, se manifiestan en desacuerdo en que su uso genera sesgos. Como ya se mencionó, este programa ve los sesgos como artefactos de los procedimientos ex- perimentales del programa rival y en consecuencia, tiene una postura relativamente optimista con respecto a la racionalidad humana. Con otras palabras, no habría acuerdo entre ambos programas en que, en general, las heurísticas tienden a producir respuestas correctas, lo que conduce a polemizar sobre nuestra aparente irracionalidad vs racionalidad. Por lo tanto, si bien la discusión sobre la rivalidad, o no, de ambos programas de investigación resulta polémica, se asume que éstos divergen con respecto a una visión optimista vs pesimista de nuestra racionalidad.

Retomando lo analizado en la sección anterior, se estima la concepción de racionalidad ecológica por sobre la concepción de racionalidad estándar puesto que ésta prepondera la adaptación del agente al contexto y con ello el éxito obtenido al momento de determinar relevancia. Tal eficiencia da cuenta de una visión optimista de la racionalidad ya que no se subrayan los errores/fallas al momento de determinar relevancia, sino que, al contrario, destaca los aciertos al que generalmente se arriba al hacerlo. Las heurísticas que promueve la noción de racionalidad ecológica al explotar las estructuras informacionales del contexto, reflejarían en mayor medida cómo es que se logran resultados adaptativos (rápidos y frugales) frente a las condiciones del contexto que condicionan (apremian) lograr la tarea encomendada. La interacción ente el agente y el contexto al que se enfrenta el agente permitiría describir cómo, pese a las limitaciones cognitivas (computacionales y temporales) se contextualiza la información utilizada logrando llevar a cabo la tarea encomendada. Es clave tener en cuenta las limitaciones reales a las que se enfrenta el agente para explicar el éxito logrado, puesto que irremediablemente se enfrenta a ellas: se quiere explicar como de hecho se selecciona exitosamente relevancia y no como se debería hacerlo; se quiere que, vistos los objetivos, las tareas llevadas a cabo resulten exitosas y no que resulten correctas o incorrectas.

Uno de los varios ejemplos (pueden verse otros en Esteban, 2004) que se pro- 
ponen para dar cuenta de las divergencias recién descriptas, es el de Gigerenzer y Selten (2001) en donde se oponen dos equipos de investigación cuya tarea es construir un robot capaz de atajar una pelota lanzada a cierta distancia de él. Esta será la tarea. El equipo I considera que como las pelotas vuelan en parábolas es menester programar todas las funciones correspondientes a todas las clases matemáticas de las parábolas; imagínese la cantidad de funciones resultantes. Al momento de seleccionar la parábola justa (relevante) el robot necesitaría poseer programas de sistemas expertos que midan la distancia del robot hasta el lugar desde donde se lanzó la pelota, la velocidad inicial y el ángulo de proyección para, de este modo, poder resolver la trayectoria y lograr el objetivo. Pero en el mundo real el viento es una variable que debe tenerse en cuenta a la hora de computar esa trayectoria. Por esta razón, debería proveer a este robot de instrumentos de medición de velocidad del viento. Pero también deberá tener en cuenta otros factores como el spin o el efecto que adquiere la bola al ser golpeada y la interacción de esos factores. Toda esta información (véase la vastedad de información disponible) debe ser computada en un intervalo entre dos y cuatro segundos que es el tiempo máximo, aproximadamente, en que la pelota está en el aire.

Por otro lado, el equipo II estudia el comportamiento eficiente de los jugadores expertos en el tema. Sobre la base de ese estudio deciden que el robot puede correlacionar solamente la variable velocidad con el ángulo de la visión. Utilizando esta heurística de la mirada el robot podrá interferir en la trayectoria de la pelota estando por sí mismo en movimiento. Este robot atiende y hace uso del ángulo de la visión (entendiéndose esto como un indicio) y no necesita (se descarta) la información que provenga de computar velocidad más dirección del viento o alguna clase de efectos como el spin. Esta heurística le permitirá estar ahí donde caiga la pelota. La heurística acotada del robot del equipo II tiene en cuenta las limitaciones del tiempo, la capacidad del cálculo y la velocidad y lo más importante, busca lograr el éxito al adaptarse a las condiciones del contexto. La heurística en cuestión hace uso de la regularidad del contexto, es rápida porque disminuye los costos temporales en complejos cómputos y sólo necesita buscar una pequeña parte de toda la información disponible.

En este ejemplo, como en el de los robots de Dennett, puede observarse que también hay un agente que indaga, entre vasta información, aquella que le sea relevante para resolver un problema teniendo en cuenta el contexto en el que se encuentra inmerso, que no es problemático por sí mismo, sino en función de lo que la tarea le requiere: desactivar una bomba o agarrar una pelota. En todos los casos se encuentran en interacción las demandas específicas acotadas de la tarea con las capacidades reales del agente que debe resolverlas. La afirmación principal de la concepción de racionalidad ecológica es que hay mecanismos de decisión que pueden explotar las estructuras informacionales del contexto donde se encuentra el agente para lograr resultados exitosos, es decir, adaptativos a ese contexto. Pero además, la concepción (optimista) de racionalidad ecológica expondría también adecuadamente por qué, la mayoría de las veces, se determina relevancia de manera eficiente, es decir cómo se comprende el alcance de los efectos secundarios de las propias acciones en tiempo real, se discriminan las implicaciones relevantes de las inferencias y se relacionan estas implicaciones con la interacción exitosa con el ambiente en un tiempo prudencial. Puesto que la clave en la comprensión de la racionalidad ecológica reside en la interacción exitosa entre el agente y su medio, se argumentará que el fenómeno fundamental del problema de marco está mejor articulado a una concepción optimista de la racionalidad humana y con ello, sería más cercano a la concepción de racionalidad ecológica propuesta por el programa de Heurísticas Rápidas y Frugales.

\section{Comentarios finales}

En este trabajo se indagó acerca de qué concepción de racionalidad, estándar o eco- 
lógica, se asocia mejor al problema de marco (considerado éste como un problema de racionalidad) o, más específicamente, cuál de ellas articularía mejor con el fenómeno fundamental del problema de marco. Así, el objetivo general de este trabajo fue indagar cómo se relaciona el problema de marco con tal debate sobre racionalidad.

Al examinar ambas concepciones de racionalidad por separado se observa que una de las principales diferencias entre ambas es la relevancia que se le adjudica a la interacción exitosa entre el agente y el contexto en el que éste se encuentra inmerso, como así también el tener o no en cuenta las limitaciones temporales/computacionales a las que el agente se enfrenta. Estos factores son primordiales para la concepción de racionalidad ecológica, puesto que el considerarlos da cuenta de cómo la mayoría de las veces se determina relevancia adecuadamente, es decir, cómo se logra con cierto éxito lograr la tarea encomendada. En contraposición, estos factores no tienen incidencia alguna para la concepción estándar de racionalidad, puesto que los criterios al que ésta adscribe están dados de antemano, no permitiendo que ningún otro factor altere el resultado. Por ello, se considera que la noción de racionalidad ecológica se encontraría mejor equipada que la noción de racionalidad estándar a la hora de exponer el aspecto psicológico del problema de marco. Con otras palabras, el concepto de racionalidad que propone el programa de Heurísticas Rápidas y Frugales satisface, en mayor medida, aquellas características de nuestra cognición que el aspecto psicológico del problema de marco pretende describir.

Resulta importante realizar una aclaración final. Que el programa de Gigerenzer y colaboradores (1996) refleje más adecuadamente aquellas características de la racionalidad del ser humano que describen mejor el aspecto psicológico del problema de marco, no significa descartar la concepción de racionalidad que defiende el programa de Tversky y Kahneman (1974). Las investigaciones típicas del programa de Heurísticas y Sesgos, y el concepto de racionalidad implícito en ellas, pueden contribuir también a la investigación sobre este aspecto, aunque sea de manera indirecta. En efecto, si bien se acepta, de acuerdo al fenómeno fundamental del problema de marco, que generalmente las personas determinan exitosamente relevancia (defendiendo así una racionalidad optimista), se reconoce también que en algunas ocasiones se cometen errores. Incluso puede haber contextos en donde se falla sistemáticamente al determinar relevancia y, en este sentido, se estaría desafiando el fenómeno fundamental del problema de marco: si se pone en duda que generalmente se determina relevancia de manera adecuada, entonces quizás el programa de Heurísticas y Sesgos podría ofrecer apoyo a esta postura. Esto no ha sido investigado en absoluto, quedando pendientes de investigación ésta $\mathrm{y}$ otras interesantes cuestiones relacionadas con el fenómeno fundamental del problema de marco.

\section{Referencias bibliográficas}

Alexander, L. \& Moore, M. (2008). Deontological Ethics. En E.N. Zalta (Ed.), The Stanford Encyclopedia of Philosophy (Invierno 2016 Ed.). Recuperado el 10 de diciembre de 2016 de http://plato.stanford. edu/entries/ethics-deontological.

Alfano, M. (2014). Expanding the Situationist Challenge to Reliabilism About Inference. En A. Fairweather (Ed.), Virtue Epistemology Naturalized: Bridges Between Virtue Epistemology and Philosophy of Science. Cham, Suiza: Springer. https://doi.org/10. 1007/978-3-319-04672-3_7

Brown, F.M. (1987). The frame problem in artificial intelligence. Proceedings of the 1987 Workshop. Los Altos: Morgan Kaufmann. https://doi.org/10.1016/B978-0-934613-323.50004-6. 
Carruthers, P. (2006). Simple heuristics meet massive modularity. En P. Carruthers, S. Laurence, \& S. Stich (Eds.), The innate mind: Culture and cognition. Oxford: Oxford University Press. https://doi.org/10. 1093/acprof:oso/9780195310139.001.0001.

Carruthers, P. (2009). An Architecture for dual Reasoning. En J. Evans \& K. Frankish (Eds.), In Two Minds: Dual Processes and Beyond. Nueva York: Oxford University Press. https://doi.org/10.1093/acprof:oso/ 9780199230167.001.0001

Crockett, L. (1994). The Turing Test and the Frame Problem: AI's Mistaken Understanding of Intelligence. New Yersey: Ablex Publishing Corporation.

Dennett, D. (1984). Cognitive Wheels: The frame problem of AI. En C. Hookway (Ed.), Minds, machines \& evolution. Cambridge: Cambridge University Press.

Dreyfus, H.L. (1979). What computers can't do: The limits of artificial intelligence. New York: Harper Colophon Books.

Esteban, J.M. (2004). La racionalidad ecológica en la teoría pragmatista del conocimiento. Acta Comportamentalia: Revista Latina de Análisis de Comportamiento, 12, 23-36.

Evans, J. \& Over, D. (1996). Rationality and reasoning. Hove (England): Psychology Press. https://doi.org/10.1080/713755785.

Fairweather, A. \& Montemayor, C. (2014). Inferential Abilities and Common Epistemic Goods. En A. Fairweather (Ed.), Virtue Epistemology Naturalized: Bridges Between Virtue Epistemology and Philosophy of Science. Cham (Suiza): Springer. https:// doi.org/10.1007/978-3-319-04672-3_8

Fodor, J. (1986). La modularidad de la mente. Madrid: Morata.

Fodor, J. (2000). The Mind Doesn't Work That Way: The Scope and Limits of Computational Psychology. Cambridge: The MIT Press.

Ford, K. \& Hayes, P. (1991). Reasoning agents in a dynamical world: the frame problem.
London: JAI Press. https://doi.org/10.2307/ 1423176.

Ford, K. \& Pylyshyn, Z. (1996). The robot's dilemma revisited: The frame problem in Artificial Intelligence. Norwood: Ablex Publishing Corporation. https://doi.org/10. 1016/0004-3702(88)90082-3.

Gigerenzer, G. (1996). On Narrow Norms and Vague Heuristics: A Reply to Kahneman and Tversky. Psychological Review, 103, 592596. http://dx.doi.org/10.1037/0033-295X. 103.3.592.

Gigerenzer, G. (2008). Rationality for Mortals: How People Cope with Uncertainty. New York: Oxford University Press. https://doi. org/10.1080/17439760.2011.614828.

Gigerenzer, G. \& Brighton, H. (2009). Home heuristics: Why Biased Minds Make Better Inferences. Topics in Cognitive Science, 1, 107-143. https://doi.org/10.1111/j.1756-765. 2008.01006.

Gigerenzer, G., Czerlinski, J. \& Martingnon L. (2002). How Good are Fast and Frugal Heuristics?. En R. Elio (Ed.), Common Sense, Reasoning and Rationality. New York: Oxford University Press. http://dx.doi. org/ 10.1093/0195147669.001.0001.

Gigerenzer, G. \& Selten, R. (2001). Bounded Rationality: The Adaptive Toolbox. Cambridge, Cambridge/MA: MIT Press.

Gigerenzer, G., Todd, P. \& the ABC Research Group (1999). Simple Heuristics That Make Us Smart. New York: Oxford University Press. https://doi.org/10.1002/acp.843.

Gilovich, T., Griffin, D. \& Kahneman, D. (2002). Heuristics and Biases: The Psycho logy of Intuitive Judgment. Cambridge: Cambridge University Press. https://doi.org/ 10.1017/CBO9780511808098.

Glymour, C. (1987). Android epistemology and the frame problem: Comments on Dennett's cognitive wheels. En Z. Pylyshyn (Ed.), The robot's dilemma: The frame problem in artificial intelligenc. Norwood: Ablex Publis 
hing Corporation. https://doi.org/10.1016/ 0004-3702(88)90082-3.

Ludwig, K. \& Schneider, S. (2008). Fodor's Challenge to the Classical computational. Theory of Mind, Mind and Language, 23, 123-143. https://doi.org/10.1111/j.1468-001 7.2007.00332.

Mc Carthy, J. \& Hayes P. (1969). Some Philosophical Problems from the Standpoint of AI. Machine Intelligence, 4, 463-502. https://doi.org/10.1016/B978-0-934613-033.50033-7.

Morgenstern, L. (1996). The problem with solutions to the frame problem. En K. Ford y P. Hayes, (Eds.), Reasoning agents in a dynamical world: the frame problem. London: JAI Press Hayes. https://doi.org/ $10.2307 / 1423176$.

Pinker, S. (2005). So How Does The Mind Works?. Mind \& Language, 20, 1-24. https://doi.org/10.1111/j.0268-1064. 2005. 00274.

Pylyshyn, Z.W. (1980).Computation and Cognition: Isssues in the Foundations of Cognitive Science. Behavioral and Brain Sciences, 3, 111-132. https://doi.org/10.1017 /S0140525X00002053.

Samuels, R. (2010). Classical Computa tionalism and the Many Problems of Cognitive Relevance. Studies in History and Philosophy of Science, 41, 280-293. https://doi.org /10.1016/j.shpsa.2010.07.006

Samuels, R., Stich, S. \& Faucher, L. (2004). Reason and rationality. En I. Niiniluoto, $\mathrm{M}$.
Sintonen \& J. Wolenski (Eds.), Handbook of Epistemology. Dordrecht: Kluwer.

Schneider, S. (2007). Yes, it does: a diatribe on Jerry Fodor's The Mind Doesn't Work, That Way. Psyche, 13, 1-15.

Shanahan, M. (1997). Solving the frame problem: A mathematical Investigation of the Common Sense Law of Inertia. Cambridge: The MIT Press.

Shanahan, M. (2009). The Frame Problem. En E.N. Zalta (Ed), The Stanford Encyclopedia of Philosophy (Invierno 2016). Recuperado el 10 de diciembre de 2016 de http:// plato.stanford.edu/archives/win2009/entries/frame-problem.

Silenzi, M.I. (2014) El problema de marco: alcances y limitaciones de los enfoques postcognitivistas. Bahía Blanca: Ediuns.

Silenzi M.I. (2015) La dualidad del problema de marco: Sobre interpretaciones y resoluciones. Tópicos: Revista de Filosofía, 47, 89-112.

Stein, L. (1990). An Atemporal Frame Problem. International Journal of Expert Systems, 3, 371-381.

Stein, E. (1996). Without Good Reason: The Rationality Debate in Philosophy and Cognitive Science. New York: Clarendon-Press. https://doi.org/10.1093/acprof:oso/9780198 237730.001 .0001$.

Tversky, A. \& Kahneman D. (1974). Judgment Judgment under Uncertainty: Heuristics and Biases. Science, 185, 1124-1131. https://doi. org/10.1126/science.185.4157.1124. 
